\title{
The Evolution of DNA Extraction Methods
}

\author{
Mariyam Dairawan ${ }^{1}$ and Preetha J Shetty ${ }^{2 *}$ \\ 1. Medical Student, College of Medicine, Gulf Medical University, UAE \\ 2. Department of Biomedical Sciences, Gulf Medical University, UAE
}

*Corresponding author: Dr. Preetha J Shetty, Associate Professor, Department of Biomedical Sciences, College of Medicine, Gulf Medical University, UAE.

To Cite This Article: Preetha J Shetty, The Evolution of DNA Extraction Methods. 2020 - 8(1). AJBSR.MS.ID.001234.

DOI: 10.34297/AJBSR.2020.08.001234.

Received: February 18, 2020; Published: 㘹 March 11, 2020

\begin{abstract}
Since the first DNA extraction performed by Friedrich Miescher in 1869, scientists have made extraordinary progress in designing extraction methods that are more reliable, easier and faster to perform, more cost-effective and produce a higher yield. The classic liquid-liquid DNA extraction method involves the use of organic and inorganic reagents such as phenol-chloroform which pose a toxic threat to humans. Many newer techniques are now based on physical extraction, which has significantly contributed to developing simpler methods for DNA handling, such as extraction using magnetic beads and cellulose-based filter paper. With the advent of gene-editing and personalized medicine, there has been an increase in the demand for reliable and efficient DNA isolation methods that can yield adequate quantities of high-quality DNA with minimal impurities. The current review addresses the evolution of different DNA extraction techniques from solvent-based methods to physical extraction methods each with its varying set of advantages and limitations.
\end{abstract}

Keywords: DNA; DNA Extraction Methods; DNA Isolation; Purification

Abbreviations: SEC: Size Exclusion Chromatography; IEC: Ion Exchange Chromatography; AC: Affinity Chromatography; DNA: Deoxyribonucleic Acid; RNA: Ribonucleic Acid; SDS: Sodium Dodecyl Sulphate; EDTA: Ethylenediaminetetraacetic Acid

\section{Introduction}

Extraction of nucleic acids is the starting point in any molecular biology study and hence is considered as a crucial process. The first crude extraction of DNA had been performed by the Swiss physician Friedrich Miescher in 1869. He had accidentally purified DNA from the nucleus while investigating proteins from leukocytes and found that the property of this substance was fundamentally different than proteins, hence coined the term "nuclein" [1]. DNA can be extracted from sources as diverse as clinical samples such as fine needle aspirates of body fluids and biopsy samples; forensic samples such as dried blood spots, buccal swabs and fingerprints; to soil, plant and animal tissue, insects, protozoa, bacteria and yeast $[2]$.

Biological information is stored in DNA through its linear sequence of polynucleotides. It is translated into mRNA and subsequently transcribed into a sequence of amino acids that determines the three-dimensional structure of the protein, which in turn determines its biological function. Hence, DNA provides the instructions to maintain biological function and is considered to be the blueprint of life [3].

At neutral $\mathrm{pH}$ and physiological salt concentration, DNA mostly exists in the B-form, the classic right-handed double helical structure. A-form nucleic acid, found in RNA-DNA and RNA-RNA duplexes, is thicker with its base pairs packed closer together due to the syn conformation of its deoxyribose sugar ring [4]. Z-DNA is present in small amounts in the cell and has a left-handed helical structure that is thinner with more base pairs per turn as compared to B-form. Its phosphodiester backbone follows a zigzag pattern owing to the alternate stacking of purine bases that maintain a syn conformation and pyrimidine bases that are rotated into the anticonformation [5].

While double-stranded DNA is present in almost all organisms, single-stranded DNA is found in very few pathogenic viruses, such as members of the families Parvoviridae, Circoviridae, Anelloviridae and Geminiviridae [6] but is also present in bacteria and cells of 
higher organisms [7].

DNA isolated from various biological samples can be used for a vast array of downstream applications, namely DNA sequencing, polymerase chain reaction (PCR), quantitative PCR (qPCR), southern blotting, random amplification of polymorphic DNA (RAPD), preparation for genomic libraries as well as amplified fragment length polymorphism (AFLP), restriction fragment length polymorphism (RFLP), short tandem repeat polymorphism (STRP), single nucleotide polymorphism (SNP) and variable number of tandem repeat (VNTR) applications [2].

In the field of medicine, these downstream applications can serve as a basis for diagnosing genetic diseases or identifying carrier status, gene therapy, pharmacogenomics to predict drug efficacy or adverse drug reactions [8] using recombinant DNA technology to derive pharmaceutical products such as hormones and vaccines [9] as well as predictive genetic testing for risk assessment and the use of targeted screening and early prevention strategies. For example, early detection of mutations in the RET proto-oncogene and pre symptomatic intervention is used to prevent the development of multiple endocrine neoplasia type 2 [10].

DNA fingerprinting is widely utilized in forensic science for identity verification of criminal suspects, paternity tests and in determining the lineage. In microbiology, nucleic acid analysis can be used to study phylogenetic relationships and help identify mutations that give rise to antibiotic resistance[10]. There are numerous other possible applications for isolated nucleic acid, but an innovation that has revolutionized research in genetics is the emergence of precise gene-editing technology, which has made significant contributions to the field of medicine, agriculture and synthetic biology [11].

The principal behind DNA extraction consists of the following steps: (1) disruption of cytoplasmic and nuclear membranes; (2) separation and purification of DNA from other components of the cell lysate such as lipids, proteins, and other nucleic acids; and (3) concentration and purification of DNA [12]. When choosing a suitable method for DNA extraction, it is crucial to ensure the quality and quantity of the isolated DNA to carry out the intended downstream applications. Other factors that should be taken into consideration to optimize the DNA extraction method include the time, cost, potential toxicities, yield, laboratory equipment and expertise requirements, as well as the required sample amount for the protocol [13]. Over a period of time different isolation methods have evolved (Figure 1) and the current review discusses the various isolation methods each with its set of advantages and disadvantages.

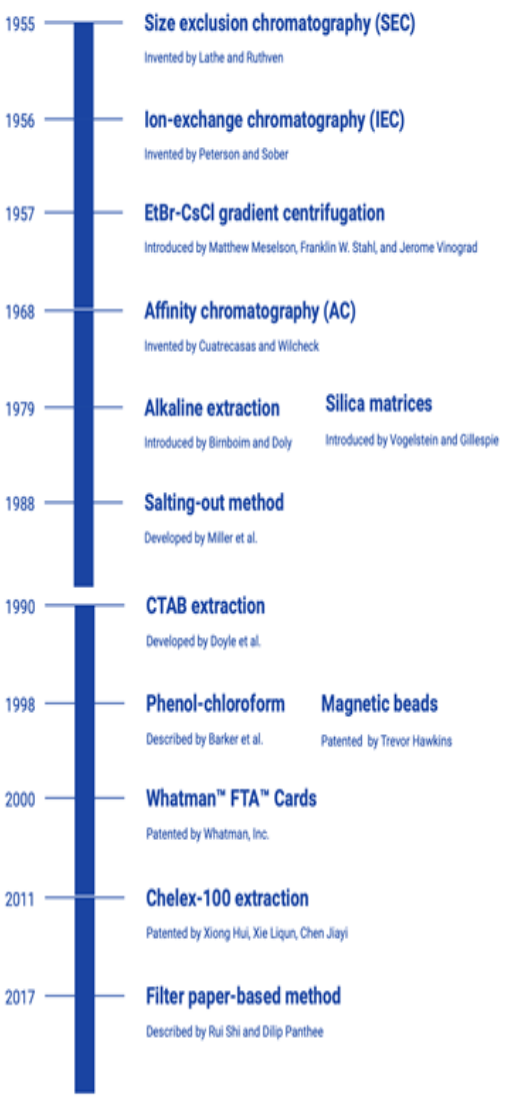

Figure 1: Timeline of the development of different DNA extraction techniques and their inventors. 


\section{Materials and Methods}

Different DNA extraction methods were searched for in electronic databases, hand searched journals and through contact with authors directly. The screening and data extraction were conducted by two reviewers independently. Electronic data sources used include PubMed, ScienceDirect, ResearchGate and Google Scholar. Main search terms used were "DNA extraction methods", "advantages of DNA extraction methods" and "limitations of DNA extraction methods", "DNA isolation methods", "DNA purification methods", "solvent-based DNA extraction" and "physical DNA extraction". Review of literature published from 1955 to till date was conducted, with a total of 116 publications reviewed.

\section{Discussion/Commentary}

\section{Chromatography-Based DNA Extraction Method}

Chromatography-based DNA extraction methods can be used to isolate DNA from any kind of biological material [14]. This method includes size exclusion chromatography (SEC), invented by Lathe and Ruthven (1955), ion-exchange chromatography (IEC) developed by Peterson and Sober (1956) and affinity chromatography (AC) reported by Cuatrecasas \& Wilcheck [15-17] In size exclusion chromatography (SEC), molecules are separated according to their molecular sizes and shape. The term gel-filtration chromatography is used when an aqueous solution is utilized to transport the DNA-containing sample through the chromatography column, as compared to gel permeation chromatography, in which an organic solvent is used instead. The column contains porous beads composed of polyacrylamide, dextran or agarose. When the sample is applied on the top and passed through the column, smaller molecules such as mRNA and proteins enter through the small pores and channels of the beads, while DNA is excluded from entering the beads and evades the matrix with its larger hydrodynamic volume. Consequently, DNA is eluted from the column faster than the smaller molecules $[18,19]$. SEC is suitable for use on substances that are sensitive to alterations in $\mathrm{pH}$ and metal ion concentrations [20].

Another DNA extraction method based on chromatography is ion-exchange chromatography (IEC). The column is first equilibrated with a solution containing DNA anion-exchange resin, which is used to selectively bind DNA with its positively charged diethylaminoethyl cellulose (DEAE) group. DNA is retained in the column while other cellular components such as proteins, lipids, carbohydrates, metabolites and RNA are eluted with medium-salt buffers. DNA can then be recovered by decreasing the $\mathrm{pH}$ or using high-salt buffers [20]. This method is relatively simple to perform when compared to other extraction methods yielding high-quality DNA, such as CsCl-gradient centrifugation [14].

Nucleic acid purification can also be achieved by affinity chromatography (AC), consisting of a similar protocol to IEC, but instead involves the use of oligo(dT) or other substances that form highly specific interactions with nucleic acid to separate it from the cell lysate [14]. However, this protocol is mainly used for the isolation of mRNA. It is time-efficient and yields a good quantity of nucleic acid [21].

\section{EtBr-CsCl Gradient Centrifugation Method}

This method was developed back in 1957 by Matthew Meselson, Franklin W. Stahl, and Jerome Vinograd [22]. DNA is first mixed with cesium chloride $(\mathrm{CsCl})$, the solution is then ultra-centrifuged at high speed $(10,000$ to $12,000 \mathrm{rpm})$ for more than 10 hours. With centrifugation, DNA separates from the rest of the substances based on its density. Depending on DNA types varying by density, one or more DNA bands appear upon reaching the isopycnic point. Ethidium bromide (EtBr) acts as an intercalating agent and is incorporated comparatively more into non-supercoiled than supercoiled DNA molecules, hence allowing supercoiled DNA to accumulate at lower densities. Location of the DNA can be easily visualized under ultraviolet light. $\mathrm{EtBr}$ and $\mathrm{CsCl}$ are removed prior to precipitation of DNA with ethanol.

This method can be used to extract DNA from bacteria, but a large amount of the material source is required. Moreover, this method is complicated, time consuming and costly due to the long duration of high-speed ultra-centrifugation required [23].

\section{Alkaline Extraction}

First introduced in 1979 by Birnboim and Doly, the method is primarily used to extract plasmid DNA from bacterial cells [24]. The sample is first suspended in an alkaline solution containing $\mathrm{NaOH}$ and SDS detergent for cell membrane lysis and protein denaturation. The alkaline condition selectively denatures chromosomal DNA which has a higher molecular weight than the intact plasmid DNA that remains double-stranded. Potassium acetate is then added to neutralize the solution, causing the chromosomal DNA to renature and precipitate from the solution. Plasmid DNA in the supernatant can be recovered after centrifugation [25]. A limitation to this method is the susceptibility for the plasmid DNA to be contaminated with fragmented chromosomal DNA.

\section{Silica Matrices}

The high affinity between silicates and DNA was first described by Vogelstein and Gillespie in 1979 [26]. This technique is based on the principle of selective binding of the negatively charged DNA with the silica surface that is covered with positively charged ions. With the DNA tightly bound to the silica matrix, the rest of the cellular contaminants can be washed out before the extracted DNA is eluted from the silica particles using distilled water or a buffer such as Tris-EDTA [27]. Various modified protocols of this technique have been described in literature, such as the use of hydrated silica matrix for DNA extraction [28]. 
The silica matrices method is simple, fast to perform, costefficient, produces high quality DNA and can be employed for automation. Commercially available kits that involve the use of silica matrices include Thermo Fisher Purelink Genomic DNA extraction kit and QIAGEN DNeasy Blood [2]. The major limitation is that the silica matrices cannot be reused as with the resins used in the anion exchange method due to their reduced binding capacity and attachment to DNA particles even after elution. However, newer methods such as maxXbond have been designed to allow multiple use of the silica matrices [29].

\section{Salting-out method}

The salting-out method is a non-toxic DNA extraction method described by Miller, Dykes, and Polesky in 1988. The DNA-containing sample is added to $3 \mathrm{~mL}$ of lysis buffer $(0.4 \mathrm{M} \mathrm{NaCl}, 10 \mathrm{mM}$ Tris- $\mathrm{HCl}$ pH 8.0 and 2 mM EDTA, pH 8.0), SDS and proteinase K. The mixture is incubated at $55-65^{\circ} \mathrm{C}$ overnight. Next, about $6 \mathrm{M}$ of saturated $\mathrm{NaCl}$ is added and the mixture is shaken for 15 seconds then centrifuged at $2500 \mathrm{rpm}$ for 15 minutes. The high salt concentration decreases protein solubility, resulting in its precipitation. The DNA-containing supernatant is pipetted into a fresh tube and can be precipitated using ethanol [16].

The salting-out method has been reported to yield high quality DNA comparable to that obtained using the phenol-chloroform method, and is superior than the latter in that it is more time efficient and cost effective and most importantly the reagents used are nontoxic [31]. It is also used to extract DNA from blood, suspension culture, or tissue homogenate [16].

\section{Cetyltrimethylammonium Bromide (CTAB) Extraction}

The CTAB extraction method was introduced by Doyle et al. in 1990 [32]. In this method, DNA-containing samples are added to $2 \%$ CTAB at alkaline $\mathrm{pH}$. In a solution of low ionic strength, the extraction buffer precipitates DNA and acidic polysaccharides from the rest of the cellular components. Solutions with high salt concentrations are then used to remove DNA from the acidic polysaccharides which form a precipitate with CTAB. Hence, this method is particularly useful for DNA extraction from plants and bacteria that produce high amounts of polysaccharides [20]. DNA is then purified using various organic solvents and alcohols, including toxic agents such as phenol and chloroform. Major limitations to this method include the use of toxic reagents and its time-consuming protocol [14].

\section{Phenol-chloroform method}

The phenol-chloroform DNA extraction method was introduced in 1998 by Barker et al. [37]. Cells are first treated with a lysis buffer containing detergents such as sodium dodecyl sulphate (SDS) to dissolve cell membranes and the nuclear envelope. Other components of the lysis buffer can include $10 \mathrm{mM}$ Tris, $1 \mathrm{mM}$ EDTA and 0.1 M NaCl. Phenol-chloroform-isoamyl alcohol (PCIA) reagent is then added in a ratio of 25:24:1 [16]. Both SDS and phenol denatures the proteins efficiently, and isoamyl alcohol prevents emulsification and hence facilitates precipitation of DNA.

The contents are mixed to form a biphasic emulsion followed by vortexing if the DNA molecules to be extracted are $<10 \mathrm{~kb}$ or by gentle shaking if DNA molecules are $10-30 \mathrm{~kb}$. The emulsion separates into two phases upon centrifugation: the upper aqueous phase composed of DNA dissolved in water and the bottom organic phase containing organic solvents and hydrophobic cellular components including proteins. The aqueous portion is then transferred to a fresh tube with the use of a pipette, and the organic phase can be discarded. Aspiration of organic solvents can be avoided by leaving behind a layer of the aqueous phase close to the interface. These steps are repeated until the interface between the aqueous and organic phases is cleared from protein [33].

Chloroform increases the density of the organic phase, preventing phenol from inverting into the aqueous phase, which might occur without the use of chloroform due to its close density to water $(1 \mathrm{~g} / \mathrm{mL})$. Hence, chloroform is used to preserve DNA from being degraded by phenol [34].

DNA can be concentrated via the standard ethanol precipitation method by adding sodium acetate solution ( $0.3 \mathrm{M}$ final concentration, pH 5.2) and ethanol in 2:1 or 1:1 ratio, followed by centrifugation to separate DNA from the solution. The pellet is washed with cold $70 \%$ ethanol to eliminate excess salt from the DNA. Finally, the DNA pellet is centrifuged again before the removal of ethanol. The pellet is dried and resuspended either in sterile distilled water or aqueous buffer [16].

The phenol-chloroform method is the gold-standard for DNA extraction. It can be used to extract DNA from blood, suspension culture, or tissue homogenate. It provides a high yield and is relatively inexpensive with a cost estimate of less than $\$ 5$ for one sample [16]. However, the toxic nature of phenol and chloroform necessitates the use of fume hoods and is a major limitation to this method. Extracted DNA samples are higher in purity than other conventional methods but lower than those obtained using columnbased methods [10]. An example of a commercially available kit based on this method is Thermo Fisher Easy-DNA® [2].

\section{SDS-Proteinase K}

Proteinase $\mathrm{K}$ is a serine protease that was first discovered by Ebeling et al. in fungus Engyodontium album in 1974 [35]. For DNA extraction, $20-50 \mu \mathrm{L}$ of $10-20 \mathrm{mg} / \mathrm{mL}$ proteinase $\mathrm{K}$ is usually added. Sodium dodecyl sulfate (SDS) is also added to dissolve the cell membrane and nuclear envelope as well as to denature and unfold proteins, exposing them to the protease activity of proteinase $\mathrm{K}$. The solution is incubated for $1-18 \mathrm{~h}$ at $50-60^{\circ} \mathrm{C}$ and can then be used to extract DNA using the phenol-chloroform or salting-out method [10]. 


\section{Silica column-based DNA extraction method}

In this method, lysis buffer (0.05 M EDTA and $3 \mathrm{ml}$ of $0.2 \mathrm{M}$ Tris, $\mathrm{pH}$ 8.0), 1\% SDS and 100 micrograms of Proteinase $\mathrm{K}$ are added to the sample. After incubation at $60^{\circ} \mathrm{C}$ for an hour, the mixture is added into a tube containing silica gel. Phenol-chloroform is added at ratio of $1: 1$ and the mixture is shaken vigorously and centrifuged for 5 minutes at $1000 \mathrm{x}$ g. This traps the proteins and organic phase beneath the silica column, while allowing the aqueous phase containing DNA to stay above the layer of gel polymer. The DNAcontaining aqueous layer is then transferred to a new tube by decanting or pipetting out and dissolved in TE buffer.

In the conventional method without silica gel, aspiration of organic solvents is usually avoided by leaving behind a layer of the aqueous phase close to the interface to prevent contamination. But with the use of silica gel, there is no interface to avoid, hence increasing the purity of the extracted DNA. Silica gel also helps prevent physical contact with the toxic reagents, and DNA yield has been reported to be $40 \%$ higher than the usual organic solventbased DNA extraction method [36].

\section{Magnetic beads}

In 1998, Trevor Hawkins filed a patent titled "DNA purification and isolation using magnetic particles" [37]. Magnetic nanoparticles coated with a DNA binding antibody or polymer with specific affinity to DNA can be employed to bind DNA to its surface. Magnetic beads are generally composed of magnetite or maghemite at the core, and surface substances that can be used include silica as well as functional groups such as sulphate and hydroxyl groups [38]. Separation of the DNA-bound magnetic beads from the cell lysate can be achieved by applying a magnetic field at the bottom of the tube using an external magnet. With the beads aggregated at the bottom of the tube, the supernatant can then be rinsed away. The magnetic pellet can be eluted via the ethanol precipitation method, after which the pellet is incubated at $65^{\circ} \mathrm{C}$ to elute the magnetic particles from the DNA [16]. The DNA yield obtained by this method is comparable to that obtained in the other conventional methods and the protocol has been shown to take less than 15 minutes to complete, which is much faster as compared to other conventional methods that can take up to several hours [39]. Furthermore, it is ideal for automation and require little equipment to perform as it does not depend on centrifugation. Another advantage over centrifugation-based methods is that this technique does not involve the use of shear forces that could damage the integrity of nucleic acids. However, this protocol is not cost effective as compared to other methods. $[14,40]$

\section{Cellulose-based paper}

Cellulose is an extensively hydroxylated polymer that has a high binding affinity for DNA. In 2000, Whatman, Inc. filed a patent titled "Ftacoated media for use as a molecular diagnostic tool" [41]. The
Whatman ${ }^{\text {TM }}$ FTA $^{\text {TM }}$ Cards are an example of commercially available cellulose-based paper that is widely used for DNA extraction. They are impregnated with buffers, detergents, and chelating agents that facilitate the extraction of DNA [42]. About 1-2 mm of the sample area is then removed using a sterilized micropunch. Before it could be processed for further down-stream applications, the punch is washed with detergent and rinsed $[14,43]$.

DNA extraction using cellulose-based paper is fast, highly convenient, does not require profound laboratory expertise and enables easy storage of the sample without refrigeration. However, downstream processing to recover pure and concentrated DNA can be very challenging [44].

\section{Chelex-100 Extraction}

In 2011, Xiong Hui, Xie Liqun and Chen Jiayi patented a DNA extraction method using chelex-100 [45]. Chelex resin is a styrenedivinylbenzene copolymer used to chelate metal ions that act as cofactors for DNases with its iminodiacetic acid groups. After overnight incubation, $5 \%$ chelex solution and proteinase $\mathrm{K}$ used to degrade the DNases are added. The tissue sample is then boiled in the $5 \%$ chelex solution to lyse the remaining membranes as well as to denature the proteins and DNA. Chelex prevents DNA from being digested by DNases that might remain after boiling, hence stabilizing the preparation. The resulting single-stranded DNA can then be concentrated from the supernatant after centrifugation [45].

Advantages of this method include the reduced risk for contamination and mis-pipetting since the procedure only involves the use of a single test tube and few manipulating steps [55]. However, the lack of purification renders this method ineffective in the removal of PCR inhibitors. Another limitation is that the isolated DNA can be unstable and is not suitable for RFLP analysis [46].

\section{Filter paper-based DNA extraction method}

In 2017, Rui Shi and Dilip Panthee describe a DNA extraction method using filter paper, which can be used to isolate DNA from plant sources. A spin plate composed of a 96-well plate (V-bottom) with a hole (about $1 \mathrm{~mm}$ in diameter) drilled into the bottom of each well is used, with each well containing a disc of Whatman ${ }^{\mathrm{TM}}$ filter paper (approximately $8 \mathrm{~mm}$ in diameter). Samples treated with lysis buffers are filtered with centrifugation. This method replaces glass fiber filters used in commercial kits for DNA extraction with filter paper, hence greatly reducing the costs of this method [47].

\section{Conclusion}

Since the first DNA extraction performed by Friedrich Miescher in 1869 , scientists have made extraordinary progress in designing extraction methods that are more reliable, easier and faster to perform, more cost-effective and produce a higher yield. Newer techniques that are more reliable and efficient have facilitated 
the advancement in knowledge about the human genome and played a major role in the advent of various fields in science such as gene-editing and personalized medicine. However, at present it seems that there is no single procedure that is applicable to all contexts of DNA extraction due to their restrictions in producing yields with optimal purity and convenience of use. Hence, solutions to overcome the limitations of these techniques are required for better results and to simplify the handling of DNA. Improvement in the design of existing methods and developing new techniques will be the driving force to direct further development of future DNA extraction technology.

\section{Acknowledgements}

College of Medicine, Gulf Medical University, Ajman, UAE.

\section{Conflict of Interest}

None.

\section{References}

1. Dahm R (2005) Friedrich Miescher and the discovery of DNA. Developmental Biology 278(2): 274-288.

2. Dhaliwal A (2013) DNA Extraction and Purification. Materials and Methods p. 3.

3. Bruce Alberts, Alexander Johnson, Julian Lewis, Martin Raff, Keith Roberts, et al. (2002) Molecular biology of the cell ( $4^{\text {th }}$ edn). Garland Science, New York, USA.

4. Hardison R (2020) 2.5: B-Form, A-Form, and Z-Form of DNA.

5. Eun H (1996) Enzymes and Nucleic Acids. Enzymology Primer for Recombinant DNA Technology, pp. 1-108.

6. Modrow S, Falke D, Truyen U, Schatzl H Viruses with a Single-Stranded DNA Genome. Molecular Virology ( $1^{\text {st }}$ edition) Springer, Berlin, Germany, pp. 875-918.

7. Popov L (1992) Single-stranded DNA from viruses, prokaryotes and eukaryotes. Molecular Biology 26(1): 25-58.

8. Reference G (2020) What is pharmacogenomics?

9. Sirkov I (2016) Nucleic Acid Isolation and Downstream Applications Nucleic Acids - From Basic Aspects to Laboratory Tools p. 1-26.

10. Evans J (2001) The complexities of predictive genetic testing. BMJ 322(7293): 1052-1056.

11. Gaj T, Sirk S, Shui S, Liu J (2016) Genome-Editing Technologies: Principles and Applications. Cold Spring Harbor Perspectives in Biology 8(12).

12. Ali N, Rampazzo R, Costa A, Krieger M (2017) Current Nucleic Acid Extraction Methods and Their Implications to Point-of-Care Diagnostics. Biomed Research International.

13. Griffiths L, Chacon Cortes D (2014) Methods for extracting genomic DNA from whole blood samples: current perspectives. Journal of Biorepository Science for Applied Medicine 2: 1-9.

14. Carpi FM, Di Pietro F, Vincenzetti S, Mignini F, Napolioni V (2011) Human DNA Extraction Methods: Patents and Applications. Recent Patents on DNA \& Gene Sequences 5(1): 1-7.

15. (1955) Proceedings of the Biochemical Society. The Biochemical journal 60(4): xxxi-xlii.

16. Peterson E, Sober H (1956) Chromatography of Proteins. I. Cellulose Ionexchange Adsorbents. Journal of The American Chemical Society 78(4): 751-755.
17. Cuatrecasas P, Wilchek M, Anfinsen C (1968) Selective enzyme purification by affinity chromatography. Proceedings of The National Academy of Sciences 61(2): 636-643.

18. Tan S, Yiap B (2009) DNA, RNA, and Protein Extraction: The Past and The Present. Journal of Biomedicine and Biotechnology: 1-10

19. Almeida A, Eusébio D, Queiroz J, Sousa F, Sousa A (2020) The use of sizeexclusion chromatography in the isolation of supercoiled minicircle DNA from Escherichia coli lysate. Journal of Chromatography A 1609.

20. Budelier K, Schorr J (1998) Purification of DNA by Anion-Exchange Chromatography. Current Protocols in Molecular Biology 42(1).

21. Chockalingam P, Jurado L, Jarrett H (2001) DNA Affinity Chromatography. Molecular Biotechnology 19(2): 189-200.

22. Meselson M, Stahl F, Vinograd J (1957) EQUILIBRIUM SEDIMENTATION OF MACROMOLECULES IN DENSITY GRADIENTS. Proceedings of The National Academy of Sciences 43(7): 581-588.

23. Leland J Cseke, Ara Kirakosyan, Peter B Kaufman, Margaret V Westfall (2012) Handbook of molecular and cellular methods in biology and medicine ( $3^{\text {rd }}$ Edition). Boca Raton: CRC Press/Taylor \& Francis Group, UK.

24. Birnboim H, Doly J (1979) A rapid alkaline extraction procedure for screening recombinant plasmid DNA. Nucleic Acids Research 7(6): 1513-1523.

25. Vogelstein B, Gillespie D (1979) Preparative and analytical purification of DNA from agarose. Proceedings of The National Academy of Sciences 76(2): 615-619.

26. Höss M, Pääbo S (1993) DNA extraction from Pleistocene bones by a silica-based purification method. Nucleic Acids Research 21(16): $3913-$ 3914.

27. Woodard D, Howard A, Down J (1993) PROCESS FOR PURIFYING DNA ON HYDRATED SILCA. United States.

28. Esser K, Marx W, Lisowsky T (2006) maxXbond: first regeneration system for DNA binding silica matrices. Nature Methods 3(1) i-ii.

29. Miller S, Dykes D, Polesky H (1988) A simple salting out procedure for extracting DNA from human nucleated cells. Nucleic Acids Research 16(3): 1215.

30. Javadi A, Shamaei M, Mohammadi Ziazi L, Pourabdollah M, Dorudinia A et al. (2014) Qualification study of two genomic DNA extraction methods in different clinical samples. Tanaffos 13(4): 41-47.

31. Doyle J (1991) DNA Protocols for Plants. Molecular Techniques in Taxonomy pp. 283-293.

32. Green M, Sambrook J (2020) Isolation of High-Molecular-Weight DNA Using Organic Solvents. CSH.

33. Elkins K (2013) DNA Extraction. Forensic DNA Biology p. 39-52.

34. Ebeling w, Hennrich N, Klockow M, Metz H, Orth H, et al. (1974) Proteinase $\mathrm{K}$ from Tritirachium album Limber. European Journal of Biochemistry 47(1): 91-97.

35. Thomas S, Moreno R, Tilzer L (1989) DNA extraction with organic solvents in gel barrier tubes. Nucleic Acids Research 17(13): 5411-5411.

36. Hawkins T (1998) DNA purification and isolation using magnetic particles. United States.

37. Elkins K (2013) Forensic DNA biology, Oxford Academic Press, Kidlington, England, pp. 43-45.

38. Saiyed Z, Ramchand C (2007) Extraction of Genomic DNA Using Magnetic Nanoparticles (Fe304) as a Solid-Phase Support. American Journal of Infectious Diseases 3(4): 225-229.

39. Abd El Aal A, Abd Elghany N, Mohamadin A, El Badry A (2010) Comparative study of five methods for DNA extraction from whole blood samples. IJHS, 3(1). 
40. Fomovskaia G, Smith M, Davis J, Jones K (2000) Fta-coated media for use as a molecular diagnostic tool, USA.

41. (2007) Whatman ${ }^{\mathrm{TM}}$ FTA $^{\mathrm{TM}}$ Cards. GE Healthcare Life Sciences.

42. (2017) Whatman® FTA® card technology.

43. Siegel C, Stevenson F, Zimmer E (2017) Evaluation and Comparison of FTA Card and CTAB DNA Extraction Methods for Non-Agricultural Taxa. Applications in Plant Sciences: 5(2).
44. Hui X, Liqun X, Jiayi C (2014) Method for rapidly extracting nucleic acid from biological sample. China.

45. Hoy M (2018) Insect molecular genetics. pp. 320.

46. Shi R, Panthee D (2017) A novel plant DNA extraction method using filter paper-based 96-well spin plate. Planta 246(3): 579-584. 